\title{
Modelling light scattering by absorbing smooth and slightly rough facetted particles
}

\author{
E. Hesse ${ }^{a}$, C.T. Collier ${ }^{a}$, A. Penttiläb, T. Nousiainen ${ }^{c}$, Z. Ulanowski ${ }^{a}$, P.H. Kaye ${ }^{a}$ \\ a University of Hertfordshire, Centre for Atmospheric and Instrumentation Research, Hatfield, \\ Hertfordshire AL10 9AB, UK \\ ${ }^{\mathrm{b}}$ Department of Physics, P.O. Box 64, University of Helsinki, Fl-00014, Finland \\ ${ }^{c}$ Finnish Meteorological Institute, Fl-00101 Helsinki, Finland
}

Keywords: light scattering, diffraction, absorbing facetted particle, rough particle

Abstract: A method for approximating light scattering properties of strongly absorbing facetted particles which are large compared to the wavelength is presented. It consists in adding the approximated external diffraction and reflection far fields and is demonstrated for a smooth hexagonal prism. This computationally fast method is extended towards prisms with slightly rough surfaces by introducing a surface scaling factor in order to account for edge effects on subfacets forming the rough surface. These effects become more pronounced with decreasing subfacet dimension to wavelength ratio. Azimuthally resolved light scattering patterns, phase functions and degree of linear polarisation obtained by this method and by the Discrete Dipole Approximation are compared for hexagonal prisms with smooth and slightly rough surfaces, respectively. 


\section{Introduction}

Airborne particles such as ice crystals [1] and Saharan dust [2] influence the Earth-atmosphere radiation balance by scattering and absorbing solar radiation. To be able to understand the radiative transfer properties of such particles, a detailed knowledge of their shapes and sizes is required. Imaging methods, e.g. [3], are widely used to obtain in situ morphological data of atmospheric particles. However, for small particles, optical aberrations, and constrained depth of field restrict the obtainable information. Such constrains do not apply to the detection of scattering patterns. Therefore, suitable detection instruments like the Small Ice Detector (SID) [4,5] have been developed. However, while conventional pattern recognition methods may be readily used to group recorded images of two-dimensional (2D) scattering patterns into broad particle shape classes [4], the inversion of the patterns required to yield quantitative morphological data is much more involved. For very complex or rough particles the presence of two-dimensional speckle can be used to derive both particle size [29] and roughness [5]. Therefore, the creation of databases of scattering patterns of known particle morphologies is extremely useful for particle characterization.

Exact methods like T-matrix [6] and semi-exact methods like the finite difference time domain (FDTD) method [8] and the discrete dipole approximation (DDA) [7] can be used for computations of light-scattering properties for non-axisymmetric particles. Those methods that are most versatile and can be applied to arbitrary particle morphologies are computationally most demanding and cannot be used if the objects are much larger than the wavelength. If singleorientation results are considered, the computational burden is reduced by several orders of magnitude. In this case, these methods can be pushed to larger sizes, as is done here for the DDA method. For objects much larger than the wavelength, approximate methods, such as the geometric optics approximation or physical optics have to be used.

In the classical geometric optics approximation, scattered light is divided into two parts, firstly light reflected or transmitted by the scatterer, and secondly externally diffracted light. Improved methods for combining the ray-tracing and diffraction parts have been presented e.g. in [9-11]. However, computational methods, which calculate the ray-tracing and diffraction contributions separately, are still widely used. In many geometric optics ray tracing codes, e.g. [1214], external diffraction is approximated by Fraunhofer diffraction on the projected cross section, applying Babinet's principle. Macke et al. [12] used the Kirchhoff approximation to model diffraction by polygonal apertures corresponding to the projected particle cross section. The method to calculate diffraction by a circular aperture at oblique incidence by means of the Kirchhoff approximation [15] has been extended to oblique incidence on polygonal apertures and applied to compute scattering patterns and phase function of absorbing facetted particles in fixed orientation [16]. (Note that due to the singular directions of rays or beams reflected from faceted particles in geometric optics, 2D scattering patterns for fixed particle orientations do not show the familiar scattering arcs but only scattering points, see e.g. [31]). In the first part of this paper we describe how polarisation treatment in [16] was improved by introducing the vector method described by Karczewski and Wolf [17]. Comparisons of phase functions, 2D scattering patterns and azimuthally averaged degree of linear polarisation for hexagonal prisms in fixed orientation are presented.

It has been reported that particle roughness can dramatically alter the scattering properties of ice crystals $[5,18,19]$. Macke et al. [12] have introduced random tilt of facets to model deviations from perfect hexagonal particle symmetry. Yang and Liou have applied a similar method to approximate polyhedral facets with Gaussian roughness [30]. Furthermore, the approach [12] has been applied to simulate rough surfaces after including Weibull statistics [18] or a normal distribution for the random tilt [20], respectively. The random tilt method has also been implemented in the Improved Geometric Optics Method (IGOM) [20,21]. However, the random tilt 
method does not model continuous surfaces. Results for a version of IGOM for randomly aligned hexagonal prisms with geometrically defined roughened surfaces have been published by Liu et al. [20]. Here, we present an approach to extend the applicability of our beam tracing method [16] to facetted particles with slightly rough surfaces by introducing a scaling factor to account for edge effects, which become more pronounced with decreasing facet to wavelength ratio. This method is tested against DDA for fixed orientation.

\section{Method}

In this section we present an improvement of the method [16] for computing scattering properties of absorbing facetted particles, in which the particle facets are treated as independent diffracting apertures. In the case of strong absorption, there are two main contributions to the scattered field: the externally diffracted field and the reflected field. These two contributions are evaluated separately using the Jones calculus (which includes phase tracing) and added. Two dimensional as well as azimuthally averaged patterns of scattered intensity and degree of linear polarisation are computed and compared with DDA results as a reference.

\subsection{External diffraction}

External diffraction is calculated using Babinet's principle. The phase shift for diffraction at an obstacle compared to diffraction through the respective aperture is $\pi$.

We consider diffraction of a plane wave at an aperture in a plane screen in vacuum. The incident wave can be presented as

$\mathbf{E}_{\mathbf{i}}(\mathbf{r})=\mathbf{E}_{\mathbf{0}} \exp \left[i k_{0}(\widehat{\mathbf{K}} \cdot \mathbf{r})\right]$

$\mathbf{H}_{\mathbf{i}}(\mathbf{r})=\mathbf{H}_{\mathbf{0}} \exp \left[i k_{0}(\widehat{\mathbf{K}} \cdot \mathbf{r})\right]$

with $\mathbf{H}_{\mathbf{0}}=\frac{1}{\mu_{0} c}\left(\widehat{\mathbf{K}} \times \mathbf{E}_{\mathbf{0}}\right)$

where $k_{0}$ is the wave number, $\widehat{\mathbf{K}}$ is the unit vector in direction of propagation and $\mathbf{r}$ the position vector at the point of observation in the far field. $\mathbf{E}_{\mathbf{i}}(\mathbf{r})$ and $\mathbf{H}_{\mathbf{i}}(\mathbf{r})$ are the incident electric and magnetic field vectors, and $\mathbf{E}_{\mathbf{0}}$ and $\mathbf{H}_{\mathbf{0}}$ are amplitude vectors, which are in general complex.

It has been shown [17] that the electric field at a point $P$ in the Fraunhofer region is given by

$\mathbf{E}(P)=\hat{\mathbf{k}} \times\left(\mathbf{F} \times \mathbf{E}_{\mathbf{0}}\right)+\mu_{0} c\left[\left(\mathbf{F} \times \mathbf{H}_{\mathbf{0}}\right)-\hat{\mathbf{k}} \cdot\left(\mathbf{F} \times \mathbf{H}_{\mathbf{0}}\right) \hat{\mathbf{k}}\right]$

where

$\mathbf{F}=\frac{i k_{0}}{4 \pi} \frac{\exp \left(i k_{0} r_{0}\right)}{r_{0}} \widehat{\mathbf{n}} \iint_{A} \exp \left[i k_{0}(\widehat{\mathbf{K}}-\hat{\mathbf{k}}) \cdot \mathbf{R}\right] d S=F \widehat{\mathbf{n}}$

Here, $\widehat{\mathbf{n}}$ is the unit normal to the plane of the aperture drawing an angle smaller than $90^{\circ}$ with the incidence direction, $\hat{\mathbf{k}}$ is a unit vector pointing from the origin of the aperture towards $P$, and $\mathbf{R}$ is the vector pointing from the origin of the aperture to the aperture point under consideration (Fig.1). 


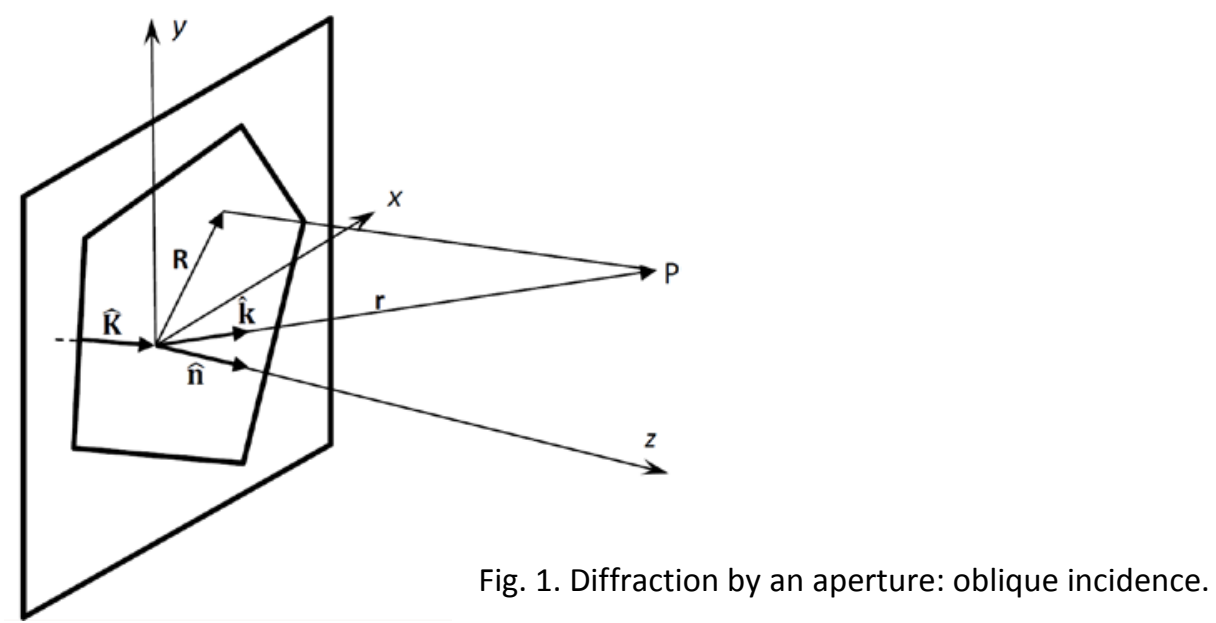

In the following, the electric and magnetic components $\mathbf{E}^{(e)}$ and $\mathbf{E}^{(m)}$, respectively, of $\mathbf{E}(P)$ are treated separately:

$\mathbf{E}^{(m)}(P)=\hat{\mathbf{k}} \times\left(\mathbf{F} \times \mathbf{E}_{\mathbf{0}}\right)$

$\mathbf{E}^{(e)}(P)=\mu_{0} c\left[\left(\mathbf{F} \times \mathbf{H}_{\mathbf{0}}\right)-\hat{\mathbf{k}} \cdot\left(\mathbf{F} \times \mathbf{H}_{\mathbf{0}}\right) \hat{\mathbf{k}}\right]$

We choose a coordinate system in such a way that the facet under consideration is positioned in the $x y$ plane, i.e. its surface normal is parallel to the $z$-axis, and the propagation vector of the incident light is contained in the $x z$-plane, which acts as a reference plane. To start, the amplitude matrix of the incident beam needs to be rotated into this reference plane by applying rotation matrix $\mathbf{R}_{\mathbf{0}}$. Rotation matrices have the general form [22]

$\mathbf{R}=\left[\begin{array}{cc}\cos \varphi & \sin \varphi \\ -\sin \varphi & \cos \varphi\end{array}\right]$

where $\varphi$ is the angle by which the initial matrix is rotated counter clockwise.

Both the incident and the diffracted field can be represented as a vector sum of one component parallel to this plane and one component perpendicular to the first vector and to the propagation vector of the wave considered. For this purpose we choose sets of unit vectors $[\hat{\mathbf{L}}, \widehat{\mathbf{M}}]$ and $[\hat{\mathbf{l}}, \widehat{\mathbf{m}}]$, where $\hat{\mathbf{L}}$ and $\hat{\mathbf{I}}$ are positioned in the reference plane, and $\widehat{\mathbf{M}}=\hat{\mathbf{L}} \times \widehat{\mathbf{K}}, \widehat{\mathbf{m}}=\hat{\mathbf{I}} \times \hat{\mathbf{k}}$. For the incident field we obtain $\mathbf{E}_{\mathbf{0}}=E_{0 L} \hat{\mathbf{L}}+E_{0 M} \widehat{\mathbf{M}}$. We can find the field components of the diffracted field parallel to $\hat{\mathbf{l}}$ and $\widehat{\mathbf{m}}$ by solving the following vector equations (expressed in $x y z$-coordinates) for $E_{l}$ and $E_{m}$

$$
\begin{aligned}
& \mathbf{E}^{(m)}=F\left\{\hat{\mathbf{k}} \times\left(\widehat{\mathbf{n}} \times\left(E_{0 L} \hat{\mathbf{L}}+E_{0 M} \widehat{\mathbf{M}}\right)\right)\right\}=E_{l}^{(m)} \hat{\mathbf{l}}+E_{m}^{(m)} \widehat{\mathbf{m}} \\
& \mathbf{E}^{(e)}=F\left\{\left[\widehat{\mathbf{n}} \times\left(\widehat{\mathbf{K}} \times\left(E_{0 L} \hat{\mathbf{L}}+E_{0 M} \widehat{\mathbf{M}}\right)\right)\right]-\hat{\mathbf{k}} \cdot\left[\widehat{\mathbf{n}} \times\left(\widehat{\mathbf{K}} \times\left(E_{0 L} \hat{\mathbf{L}}+E_{0 M} \widehat{\mathbf{M}}\right)\right)\right] \hat{\mathbf{k}}\right\}=E_{l}^{(e)} \hat{\mathbf{l}}+E_{m}^{(e)} \widehat{\mathbf{m}}
\end{aligned}
$$

and obtain

$$
E_{l}^{(m)}=F\left[-E_{0 L} K_{z} \frac{\sqrt{1-k_{y}^{2}}}{\sqrt{1-K_{y}^{2}}}+E_{0 M}\left(k_{x} k_{y} \frac{\sqrt{1-K_{y}^{2}}}{\sqrt{1-k_{y}^{2}}}-K_{x} K_{y} \frac{\sqrt{1-k_{y}^{2}}}{\sqrt{1-K_{y}^{2}}}\right)\right]
$$




$$
\begin{aligned}
& E_{m}^{(m)}=-F E_{0 M} k_{z} \frac{\sqrt{1-K_{y}^{2}}}{\sqrt{1-k_{y}^{2}}} \\
& E_{l}^{(e)}=-F E_{0 L} k_{z} \frac{\sqrt{1-K_{y}^{2}}}{\sqrt{1-k_{y}^{2}}} \\
& E_{m}^{(e)}=F\left[E_{0 L}\left(K_{x} K_{y} \frac{\sqrt{1-k_{y}^{2}}}{\sqrt{1-K_{y}^{2}}}-k_{x} k_{y} \frac{\sqrt{1-K_{y}^{2}}}{\sqrt{1-k_{y}^{2}}}\right)-E_{0 M} K_{z} \frac{\sqrt{1-k_{y}^{2}}}{\sqrt{1-K_{y}^{2}}}\right]
\end{aligned}
$$

To obtain the components into directions $\mathbf{1}$ or $\widehat{\mathbf{m}}$ of the diffracted electromagnetic field, eqs. (8a) and $(8 c)$, or (8b) and (8d), have to be added, respectively.

$$
\begin{aligned}
& E_{l}^{(e, m)}=F\left[-E_{0 L}\left(k_{z} \frac{\sqrt{1-K_{y}^{2}}}{\sqrt{1-k_{y}^{2}}}+K_{z} \frac{\sqrt{1-k_{y}^{2}}}{\sqrt{1-K_{y}^{2}}}\right)+E_{0 M}\left(k_{x} k_{y} \frac{\sqrt{1-K_{y}^{2}}}{\sqrt{1-k_{y}^{2}}}-K_{x} K_{y} \frac{\sqrt{1-k_{y}^{2}}}{\sqrt{1-K_{y}^{2}}}\right)\right] \\
& E_{m}^{(e, m)}=F\left[E_{0 L}\left(K_{x} K_{y} \frac{\sqrt{1-k_{y}^{2}}}{\sqrt{1-K_{y}^{2}}}-k_{x} k_{y} \frac{\sqrt{1-K_{y}^{2}}}{\sqrt{1-k_{y}^{2}}}\right)-E_{0 M}\left(K_{z} \frac{\sqrt{1-k_{y}^{2}}}{\sqrt{1-K_{y}^{2}}}+k_{z} \frac{\sqrt{1-K_{y}^{2}}}{\sqrt{1-k_{y}^{2}}}\right)\right]
\end{aligned}
$$

These equations can be expressed in matrix form: $\left[\begin{array}{c}E_{l}^{(e, m)} \\ E_{m}^{(e, m)}\end{array}\right]=\mathbf{D}\left[\begin{array}{c}E_{0 L} \\ E_{0 M}\end{array}\right]$ where the amplitude

$$
\text { diffraction matrix } \mathbf{D}=F\left[\begin{array}{cc}
-\left(k_{z} \frac{\sqrt{1-K_{y}^{2}}}{\sqrt{1-k_{y}^{2}}}+K_{z} \frac{\sqrt{1-k_{y}^{2}}}{\sqrt{1-K_{y}^{2}}}\right) & \left(k_{x} k_{y} \frac{\sqrt{1-K_{y}^{2}}}{\sqrt{1-k_{y}^{2}}}-K_{x} K_{y} \frac{\sqrt{1-k_{y}^{2}}}{\sqrt{1-K_{y}^{2}}}\right) \\
\left(K_{x} K_{y} \frac{\sqrt{1-k_{y}^{2}}}{\sqrt{1-K_{y}^{2}}}-k_{x} k_{y} \frac{\sqrt{1-K_{y}^{2}}}{\sqrt{1-k_{y}^{2}}}\right) & -\left(K_{z} \frac{\sqrt{1-k_{y}^{2}}}{\sqrt{1-K_{y}^{2}}}+k_{z} \frac{\sqrt{1-K_{y}^{2}}}{\sqrt{1-k_{y}^{2}}}\right)
\end{array}\right]
$$

In the next step, by multiplying with a rotation matrix $\mathbf{R}_{\mathbf{1}}$ the matrix $\mathbf{D}$ is rotated around the vector $\hat{\mathbf{k}}$ into the scattering plane containing the incidence vector $\widehat{\mathbf{K}}$. Finally, the pre-multiplication matrix $\mathbf{R}_{\mathbf{p}}$ $[12,22]$ is included to rotate the amplitude matrix of the incident beam around its propagation vector into the plane containing $\hat{\mathbf{k}}$. Therefore, the amplitude matrix describing external diffraction by the particle into direction $\hat{\mathbf{k}}$ can be written as $\mathbf{S}_{\mathbf{D}}=\mathbf{R}_{\mathbf{1}} \cdot \mathbf{D} \cdot \mathbf{R}_{\mathbf{0}} \cdot \mathbf{R}_{\mathbf{p}}$.

\subsection{External reflection}

Next, external reflection of the incident plane wave by the crystal facets facing it is considered. As a first step, the amplitude matrix of the incident wave is rotated by rotation matrix $\mathbf{R}_{\mathbf{0}}$ into the plane containing the surface normal and the direction of propagation of the incident wave. Next, we apply the generalised Fresnel amplitude reflection matrix 


$$
\mathcal{R}=\left[\begin{array}{cc}
\frac{\tilde{n}^{2} \cos \vartheta_{i}-\sqrt{\tilde{n}^{2}-\sin ^{2} \vartheta_{i}}}{\tilde{n}^{2} \cos \vartheta_{i}+\sqrt{\tilde{n}^{2}-\sin ^{2} \vartheta_{i}}} & 0 \\
0 & \frac{\cos \vartheta_{i}-\sqrt{\tilde{n}^{2}-\sin ^{2} \vartheta_{i}}}{\cos \vartheta_{i}+\sqrt{\tilde{n}^{2}-\sin ^{2} \vartheta_{i}}}
\end{array}\right]
$$

where $\tilde{n}$ is the complex refractive index and $\vartheta_{i}$ is the incidence angle $[12,23]$. The $(1,1)$ and $(2,2)$ elements of $\mathcal{R}$ are the amplitude reflection coefficients for the electric field parallel and perpendicular to the incidence plane, respectively. Far field diffraction of the reflected wave into direction $\hat{\mathbf{k}}$ is calculated in the same way as described above for externally diffracted light by applying the diffraction matrix $\mathbf{D}$ (the surface normal $\widehat{\mathbf{n}}$ is drawing an angle smaller than $90^{\circ}$ with the geometric optics reflection vector). Finally, the amplitude matrix is rotated into the plane containing the propagation vector of the incident wave by applying $\mathbf{R}_{\mathbf{1}}$. Again, the pre-multiplication matrix $\mathbf{R}_{\mathrm{p}}$ is included to rotate the amplitude matrix of the incident beam around its propagation vector of into the plane containing $\hat{\mathbf{k}}$. The amplitude phase matrix describing external reflection into direction $\hat{\mathbf{k}}$ by the particle can be written as $\mathbf{S}_{\mathbf{R}}=\mathbf{R}_{\mathbf{1}} \cdot \mathbf{D} \cdot \mathcal{R} \cdot \mathbf{R}_{\mathbf{0}} \cdot \mathbf{R}_{\mathbf{p}}$.

For each direction $\hat{\mathbf{k}}$ the amplitude matrices due to external reflection and diffraction are added. Using the definition of the Stokes vector, the elements of the scattering matrix are obtained from the amplitude matrix [24].

\section{Results and discussion}

\subsection{Hexagonal prism with a smooth surface}

In the following, results obtained with the above method for an isometric hexagonal prism with an edge length of the basal facets of $5 \mu \mathrm{m}$, a prism height of $10 \mu \mathrm{m}$ and a refractive index $n=1.31+0.1 i$ illuminated by light of $500 \mathrm{~nm}$ wavelength (size parameter $20 \pi$ ) are compared with DDA results. Due to the large imaginary part of the refractive index, most light that enters the crystal will be absorbed. Therefore, for simplicity, the refracted component is excluded and only external reflection and diffraction are considered. The hexagonal prism is aligned in such a way that the prism axis is tilted by $30^{\circ}$ with respect to the direction of incidence (see inset of Fig. 3 ). The plane defined by the prism axis and the direction of incidence contains also two prism facet edges. Fig. 2 shows the azimuthally resolved distribution of the $(1,1)$ element of the scattering matrix, $P_{11}$, obtained from classical geometric optics, i.e. ray-tracing combined with diffraction at projected cross section (left data column) and beam tracing (difference of the amplitude matrices of external reflection and diffraction, middle data column) compared with the corresponding DDA results (right column). The geometric optics (GO) peaks due to reflection can be seen as singular points in the first data column. Reflection peaks due the two prism facets are positioned at $\left[\vartheta=51.3^{\circ}, \varphi=56.3^{\circ}\right]$ and $\left[\vartheta=51.3^{\circ}\right.$, $\varphi=123.7^{\circ}$ ], respectively, where $\vartheta$ and $\varphi$ are the elevation and azimuthal angles, respectively. Reflection at the basal facet causes a GO peak at $\left[\vartheta=120^{\circ}, \varphi=270^{\circ}\right]$. Since the projected cross section is perpendicular to the incident beam, the external diffraction arcs centred the direct forward direction are straight. In contrast to this, the beam tracing result shows external diffraction and reflection arcs bent according to the orientation of the corresponding facets with respect to the direction of incidence or reflection, respectively. Note that the external diffraction arcs, unlike for the classical GO model, pass directly through the external reflection maxima and overlap with the corresponding reflection arcs. 

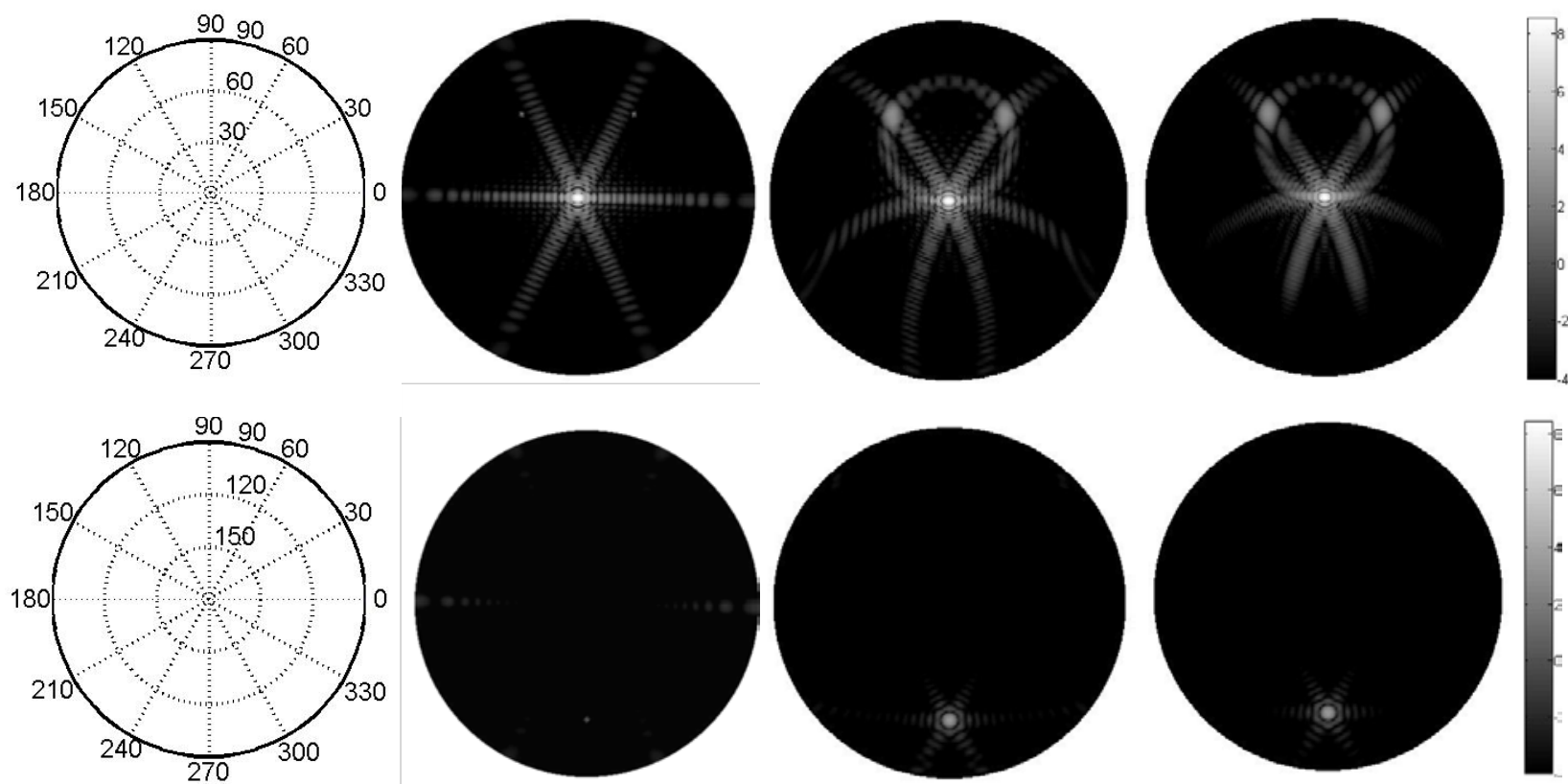

Fig. 2. Azimuthally resolved $P_{11}$ : classical geometric optics (ray-tracing combined with diffraction at projected cross section (left data column), beam tracing (middle data column) and DDA results (right column) for an isometric, hexagonal, absorbing prism with a size parameter of $20 \pi$. The top and bottom row show scattering into the forward and back scattering hemisphere, respectively.

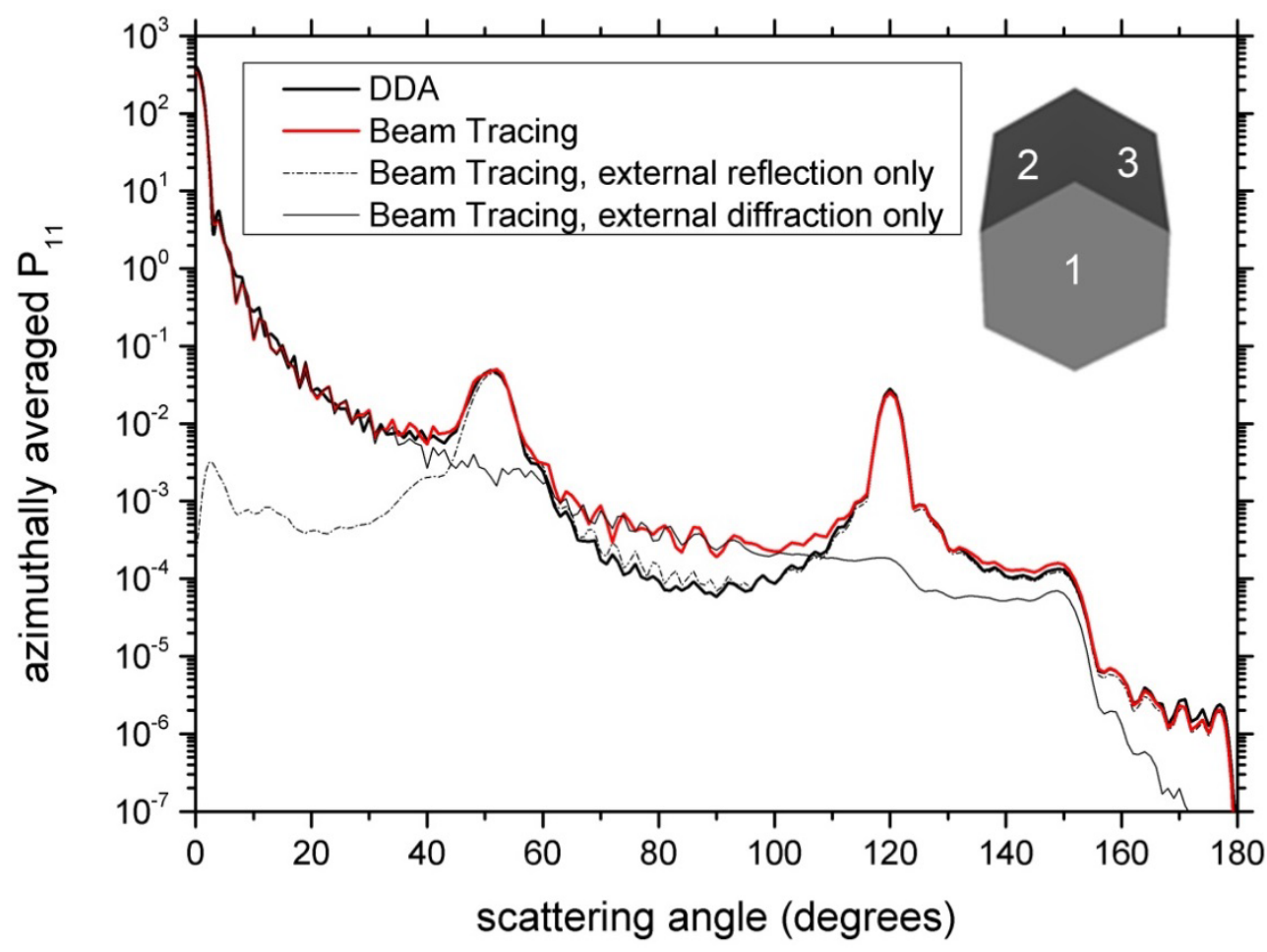

Fig. 3. Comparison of the azimuthally averaged phase functions computed by the DDA and the beam tracing method. For the latter case, results are also shown for external diffraction or external reflection only.

The beam tracing results agree quite well with the DDA data. However, the three radial arcs centred at the direct forward direction have higher intensities towards side scattering in the beam tracing than in the DDA plots. This indicates that external diffraction is too strong. Fig. 3 shows the corresponding phase functions averaged over azimuth angles and separate graphs of the contributions due to external diffraction only and reflection only. The main contribution between $0^{\circ}$ 
and $40^{\circ}$ is due to external diffraction, whereas for larger scattering angles external reflection seems to dominate. The overall beam tracing result is too high in the angular region between about $60^{\circ}$ and $110^{\circ}$ due to external diffraction being too strong. This is likely to be due to computing diffraction at apertures or facets within infinite target planes. In this way the three-dimensionality of the object (or synonymously, its edge effects) are not fully taken into account. This inaccuracy will be greater for smaller surfaces and for scattering into directions further away from the forward scattered signal (see analogous discussion for scalar diffraction theory in [32]).

Fig. 4 shows the azimuthally resolved degree of linear polarisation (DLP) computed from beam tracing and from DDA (right column). For explanatory purposes we give also results which take account of external reflection only (leftmost column). The external reflections are linked to positively polarised features: in Fig. 4, the two prism facets cause the two positively polarised diagonal arcs in the forward scattering hemisphere, which extend into the backscattering hemisphere; Fig. 5 shows beam tracing results for one prism facet only. The DLP is stronger for the reflections from the two prism facets than from the basal facet, because the incidence angle of $64.35^{\circ}$ at the prism facets is closer to the pseudo-Brewster angle [25] of 50.3 than the incidence angle of $30^{\circ}$ at the basal facet see Fig. 6 for beam tracing results for basal facet only. The second and third column of Figs. 4 to 6 show the DLP obtained after adding diffraction and reflection intensities (i.e. not taking phase differences into account) or subtracting (due to phase shift of $\pi$ ) diffraction from reflection amplitude, respectively. Taking care of the respective phases of external diffraction and reflection by combining their amplitudes is essential for a better agreement with the DDA data. This applies particularly to the backscattering hemisphere in Fig. 3, where only these results show the diffraction arcs of the $\left[\vartheta=120^{\circ}, \varphi=270^{\circ}\right]$ reflection (the horizontal arc is most easily recognisable). The region $90^{\circ} \leq \vartheta \leq 120^{\circ}$ contains a distribution of positive DLP regions, even though overall DLP is weaker than in the DDA results. The most likely reason for the disagreement between the DLP results obtained from DDA and beam tracing is the assumption of infinite target planes when computing diffraction at facets, i.e. the neglect of edge effects. Also, there might be small contributions from refraction and higher order reflection events which have not been taken account of in the current beam tracing implementation.
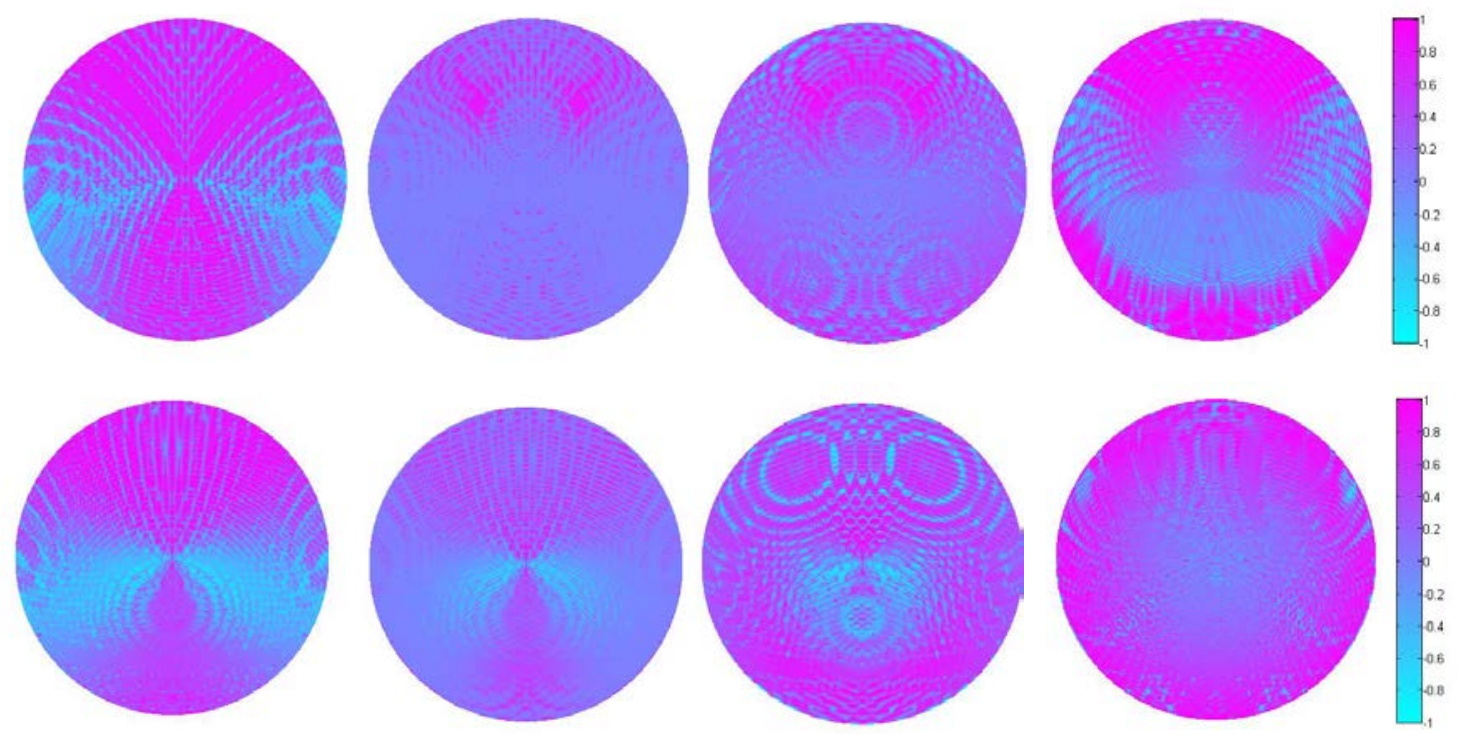

Fig. 4. 2D scattering patterns showing the degree of linear polarisation: beam tracing: external reflection only ( $1^{\text {st }}$ column), external reflection and diffraction added as intensities ( $2^{\text {nd }}$ column), and beam tracing with diffraction amplitude subtracted from external reflection amplitude ( $3^{\text {rd }}$ column), DDA (4th column). The top and bottom row show scattering into the forward and backscattering hemisphere, respectively. 

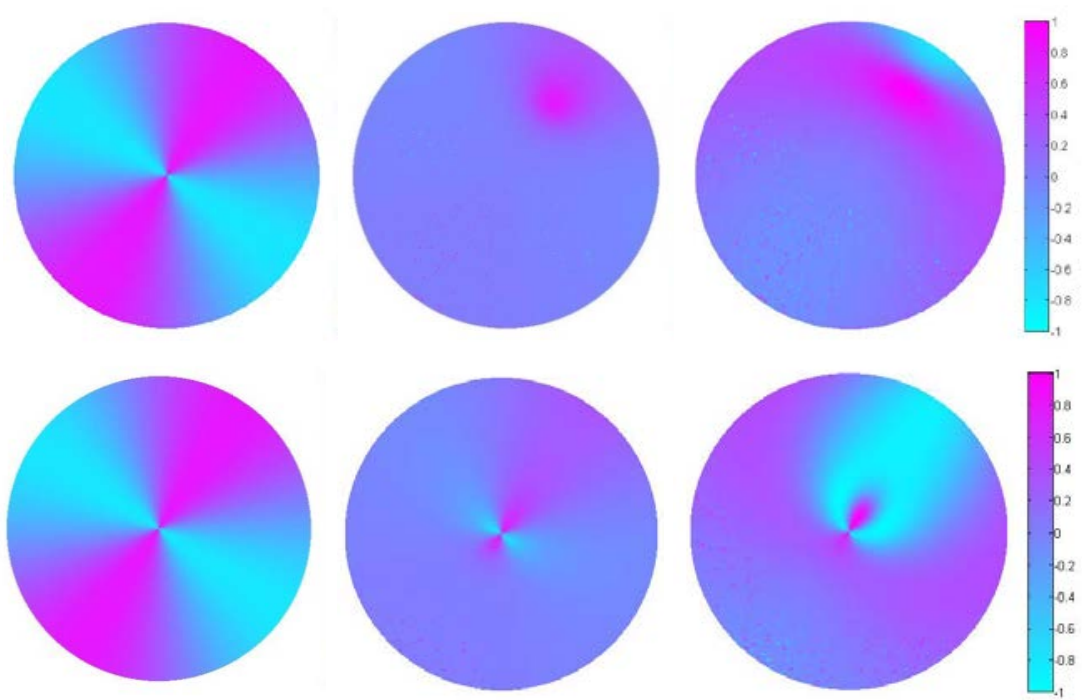

Fig. 5. Degree of linear polarisation for the right-hand, upward facing prism facet only (see inset of Fig. 2): reflection only (left column), reflection and diffraction intensities added (middle column), diffraction amplitude subtracted from external reflection amplitude (right column).
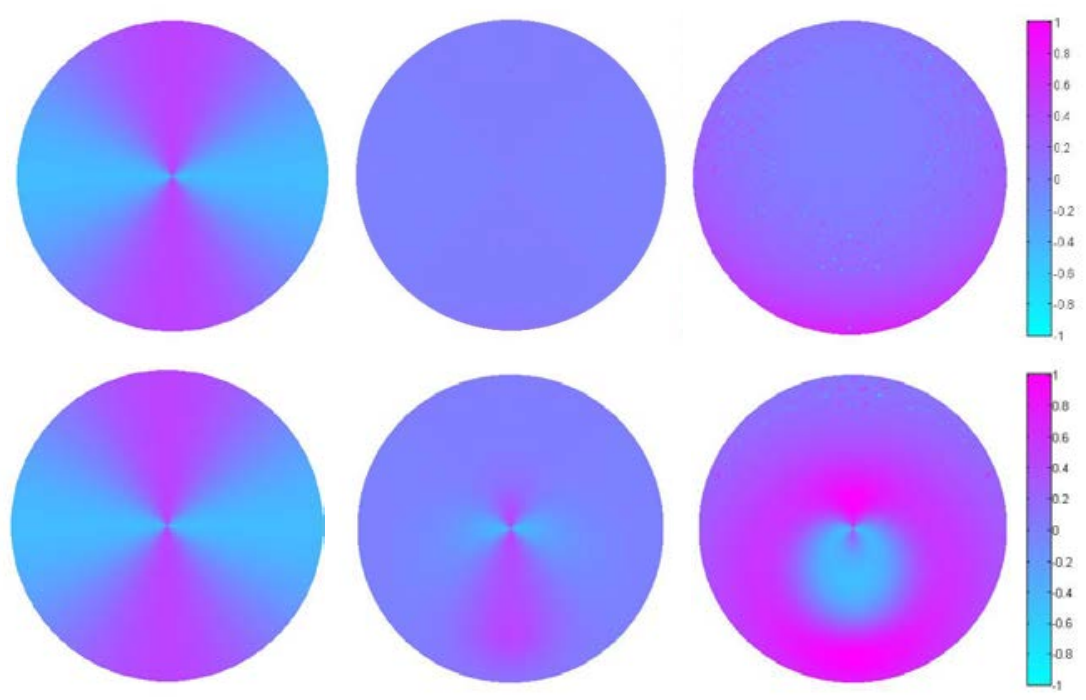

Fig. 6. Degree of linear polarisation for the incident beam facing basal facet only: reflection only (left column), reflection and diffraction intensities added (middle column), diffraction amplitude subtracted from external reflection amplitude (right column). 
Fig. 7 shows the azimuthal average of $\operatorname{DLP} \sum_{\varphi=1^{\circ}}^{360^{\circ}}\left[-P_{12}(\vartheta, \varphi) / P_{11}(\vartheta, \varphi)\right] / 360$ as a function of the scattering angle for beam tracing and DDA results. The dashed and solid thin lines correspond to the second and third columns of Figs. 4 to 6, respectively. The figure demonstrates how the combination of the externally reflected and diffracted wave amplitudes produces DLP values which could not be obtained from the externally reflected component alone. We find good agreement of beam tracing with DDA in the scattering angle regions from zero to about $63^{\circ}$ and from about $112^{\circ}$ to $180^{\circ}$. The absolute value of $-P_{12} / P_{11}$ is too low in the region between $63^{\circ}$ and $112^{\circ}$, most likely because the external diffraction contribution in beam tracing is too strong. One should note that scattered intensity in this angular region is quite low. Therefore, small absolute errors in $P_{11}$ and $P_{12}$ can cause fairly large errors in DLP.

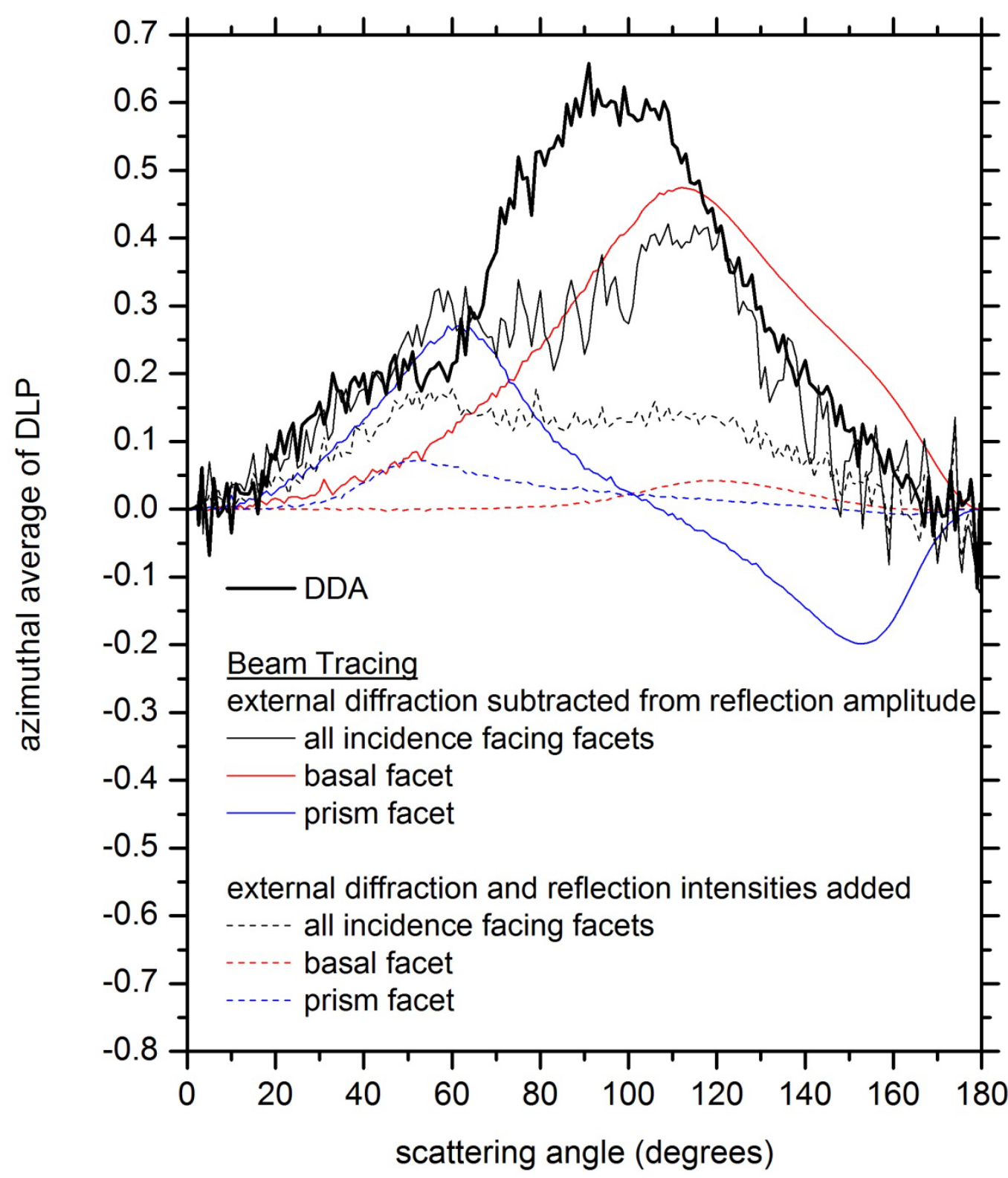

Fig. 7. Azimuthally averaged degree of linear polarisation. 


\subsection{Hexagonal prism with slightly rough surface}

So far, light scattering by a polyhedron with smooth surfaces has been considered. In the following we investigate the effect of surface roughness. Since it is thought that Gaussian random surfaces $[26,27]$ are a good model for roughness occurring on ice crystals in the atmosphere [5], we choose this type of roughness for our model particle.

Fig. 8 shows a comparison of DDA results for the dependence of scattered intensity on scattering angle for a smooth and a slightly rough prism. Both of them have an edge length of basal facets of $5 \mu \mathrm{m}$ and a column height of $10 \mu \mathrm{m}$, as before. The rough prism has a Gaussian random surface with a correlation length of $0.5 \mu \mathrm{m}$ and a standard deviation of $0.1 \mu \mathrm{m}$. The particle orientation is the same as in the previous section (see insets of Fig. 8). Since the standard deviation of the surface

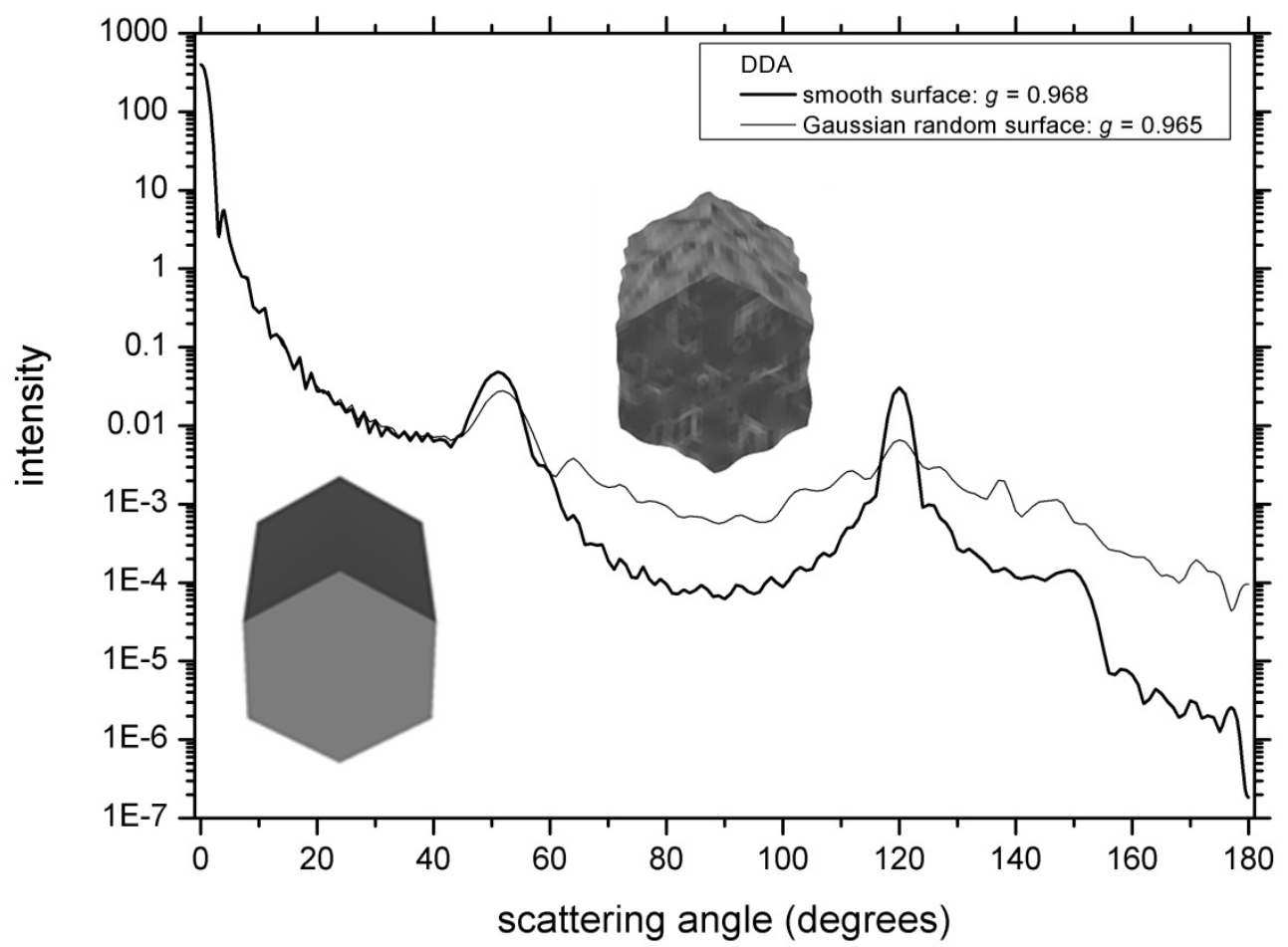

Fig. 8. Azimuthally averaged phase functions for a smooth and a rough hexagonal prism (roughness correlation length $0.5 \mu \mathrm{m}$, standard deviation $0.1 \mu \mathrm{m}$, average subfacet dimension to wavelength ratio for the basal facet 0.412 ) computed using DDA.

roughness is much smaller than the wavelength, the modification of the (three-dimensional) outline compared to the smooth crystal can be neglected when calculating external diffraction. Therefore, external diffraction is in the following modelled as diffraction by the corresponding smooth prism. Forward scattering up to about $40^{\circ}$ is dominated by external diffraction occurring at the outline of the crystal.

As can be expected, the reflection peaks are reduced in intensity compared to the smooth crystal, and light is scattered into wider angular ranges. This effect becomes stronger with increasing scattering angle and is most pronounced for the $120^{\circ}$ peak, which is the reflection peak furthest away from the incidence direction. (Due to the strong absorption, the asymmetry parameter $g$ is affected mainly by external diffraction and has a value of 0.97 for both the smooth and the rough prism.) Reflection by the rough upward facing facets was initially modelled by adding reflection 
amplitudes due to the individual subfacets. This resulted in completely spread out reflection peaks. However, the DDA result clearly shows a pronounced peak at $120^{\circ}$, which is similar in width to the corresponding peak for the smooth prism. Because the standard deviation of the roughness is only a fraction of the wavelength, the light scattering pattern still shows features of the underlying smooth surface. This discrepancy between the initial beam tracing and the DDA results is most likely due to regarding the subfacets as independent apertures, in the same way as the facets of the smooth crystal were treated in section 3. Because the subfacet dimension to wavelength ratio of 0.41 is much smaller than the facet to wavelength ratio of 10 in the smooth crystal case, the disagreement with the DDA results is much larger. To correct for these edge effects, a model crystal with flatter surfaces was used for the light scattering computations. (For a completely flat facet, line integral contributions for the same edge shared by two subfacets will cancel each other entirely, and only the integral contributions for non-shared edges, i.e. the outer facet edges, remain.) A scaling factor $k$ with $0<k<1$ was introduced: the distance $h_{0}$ of a vertex from the average crystal plane (corresponding to the smooth crystal) was calculated, and the new vertex was set to $h=k h_{0}$. The scaling factor has a limit of one when approaching smooth surfaces and decreases with increasing average angle between the normals of neighbouring subfacets (in the following called inter-subfacet angle $\alpha$ ). The value of $k$ was obtained by best fits against DDA intensity vs. scattering angle data. Fig. 9b shows graphs of $\sin (\alpha)$ vs. $d / \lambda$, where $d$ is the average subfacet dimension (i.e. the square root of its area), with three different subfacet sizes for the same Gaussian random surface on the respective facet. The average $d / \lambda$ ratios for the basal facet $d_{b}$ are $0.103,0.206$ or 0.412 (see Figs. 9a). Due to the different grids for basal facets and prism facets, these ratios are slightly shifted for the prism facets (for the basal facet, the subfacets are equilateral triangles when projected into the main facet plane, whereas for the prism facets they are right-angle triangles obtained by bisecting squares). Because of the method used for creating the hexagonal prism from a Gaussian random plane, the basal facet is slightly smoother than the prism facets. This causes $\sin (\alpha)$ to grow less with increasing $d / \lambda$ than it does for the prism facets. To quantify this, best fits $\sin (\alpha)=c_{2}(d / \lambda)^{\mathrm{c}_{1}}$ were introduced for crystals with the same correlation length and standard deviation but different $d / \lambda$. For the basal facet facing the incident light we obtained $c_{1}=0.698$. The averaged value $c_{1}$ for the two prism facets exposed to the incident light is 0.842 . The scaling factor $k$ is set to be inversely proportional to $c_{1}$, which means that scaling affects smoother surfaces less than rougher ones. For facet 1 , a value $k=0.223$ was obtained, and $k=0.185$ for facets 2 and 3 .

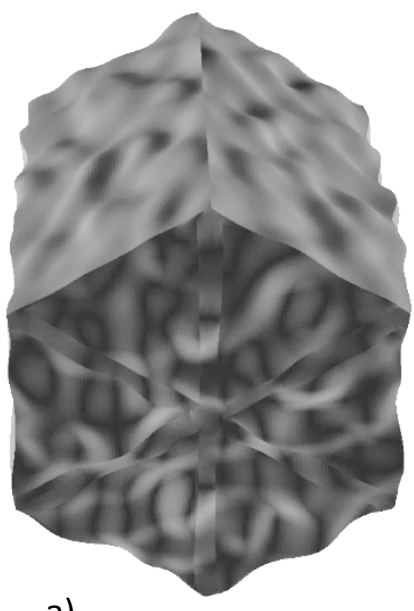

a)

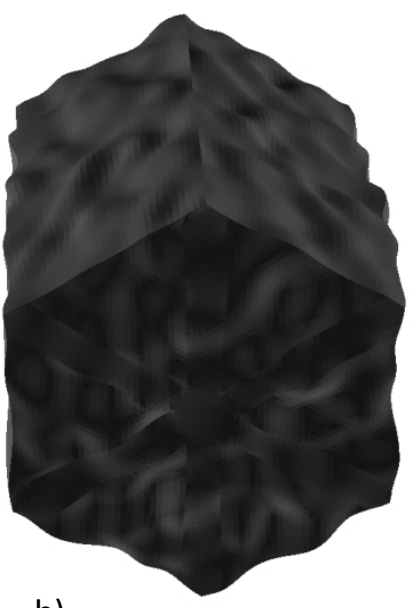

b)

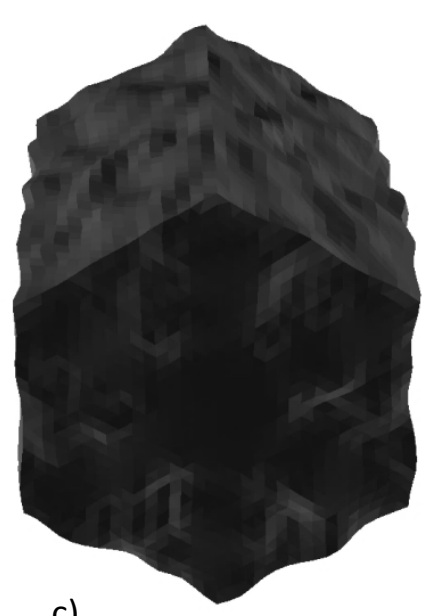

c)

Fig. 9a. Hexagonal column with Gaussian random surface (correlation length $0.5 \mu \mathrm{m}$, standard deviation $0.1 \mu \mathrm{m}$ ). The average subfacet dimension to wavelength ratio for the basal facet $d_{b}$ is a) $\left.0.103, b\right) 0.206$ and c) 0.412 . 


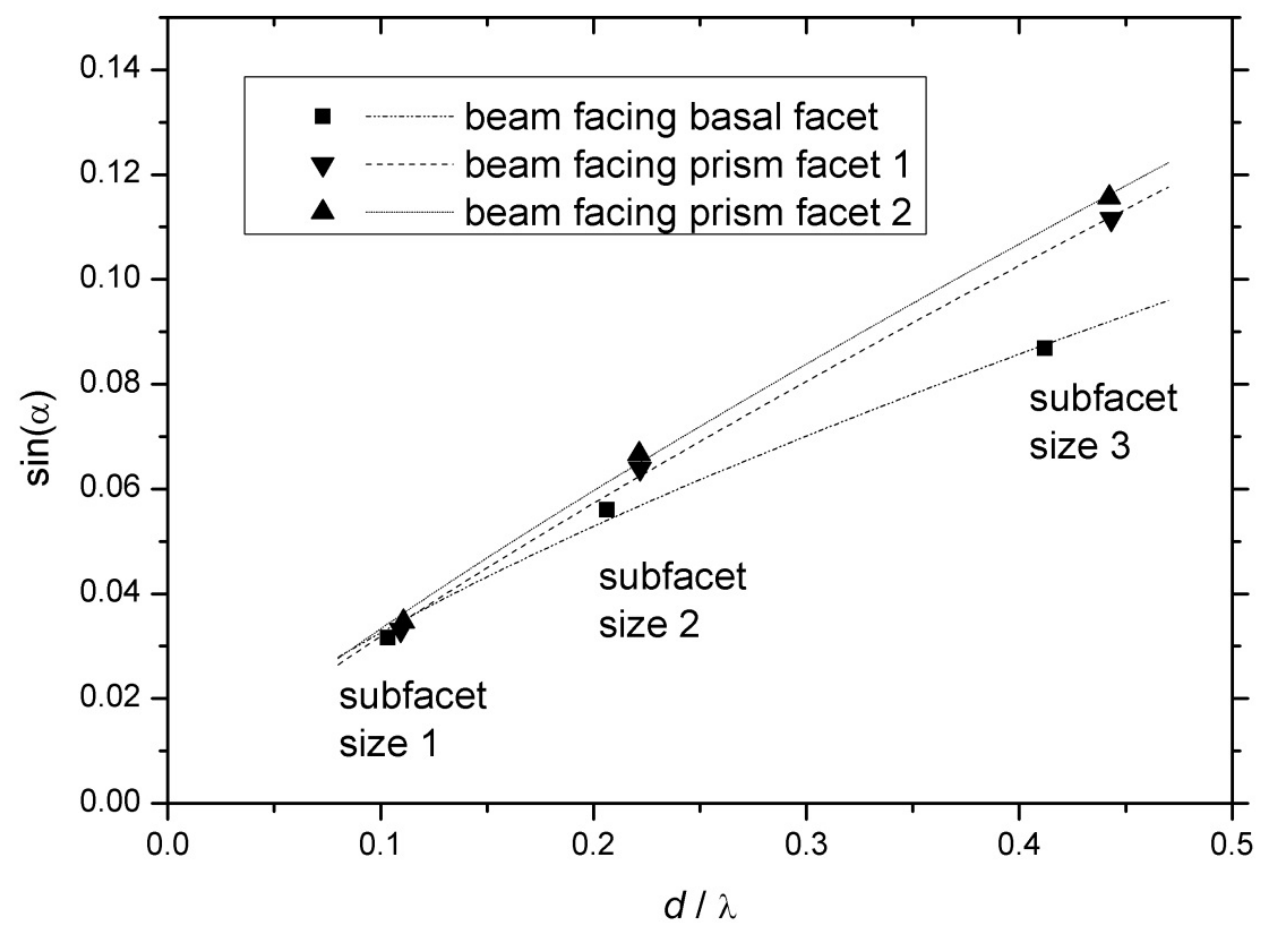

Fig. 9b. Plot of (average subfacet dimension $d$ /wavelength $\lambda$ ) vs. sine(average inter subfacet angle $\alpha$ ) for hexagonal column with Gaussian random surface (correlation length $0.5 \mu \mathrm{m}$, standard deviation $0.1 \mu \mathrm{m}$ ).

Fig. 10 shows azimuthally averaged $P_{11}$ vs. scattering angle plots computed with DDA and the beam tracing method, using the scaling factors $k$ described above, for the three different subfacet sizes. Both the DDA and the beam tracing results show slightly less sideward scattering and less widening of the intensity distribution around $120^{\circ}$ for larger than for smaller subfacet sizes. This trend agrees with results by Kahnert et al. [28], who found decreased side and backscattering compared to spheres and spheroids for absorbing high order Chebyshev particles, which were considered as proxy for small-scale surface roughness (here, the larger facetted particles deviate more from the Gaussian random surface than smaller facetted ones). For scattering angles lower than $60^{\circ}$ and close to $120^{\circ}$, i.e. in the regions dominated by external diffraction and the external reflections at $51^{\circ}$ and $120^{\circ}$, there is good agreement of beam tracing and DDA results. However, side and backscattering are too high, again, most likely due to modelling diffraction as occurring on facets (i.e. the corresponding apertures) within infinite target planes, not taking the three-dimensionality of the object fully into account. The azimuthally resolved distributions of $P_{11}$ obtained from DDA and beam tracing are presented in Fig. 11 for an average basal subfacet dimension of $0.412 \lambda$. DDA and beam tracing show similar speckle patterns. 


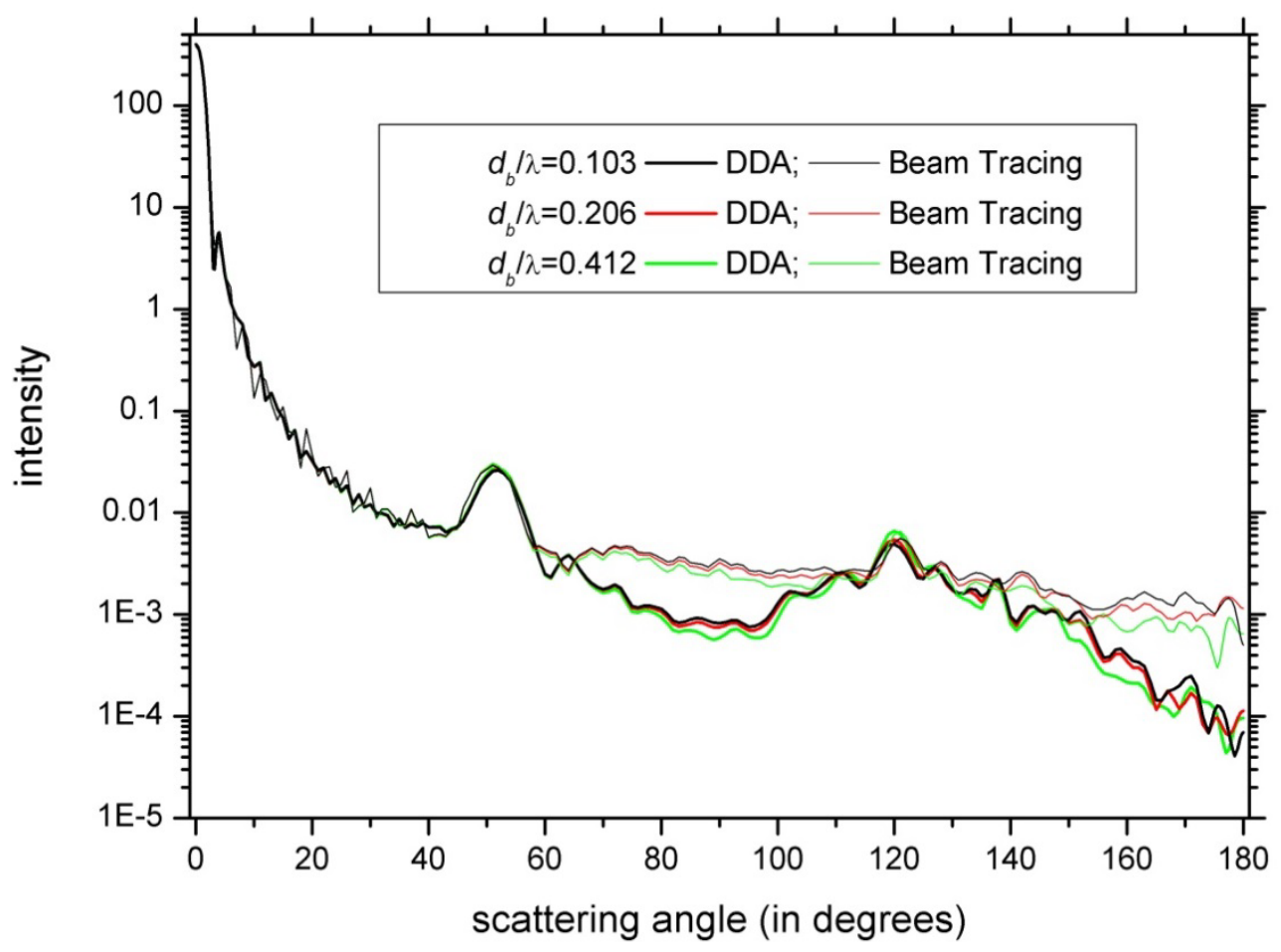

Fig. 10. Scattered intensity computed by DDA and beam tracing (using the scaling factors $k$ described in the text) for a hexagonal prism with Gaussian random surface (correlation length $0.5 \mu \mathrm{m}$, standard deviation $0.1 \mu \mathrm{m}$ ) modelled for three different subfacet sizes $d_{b}$.
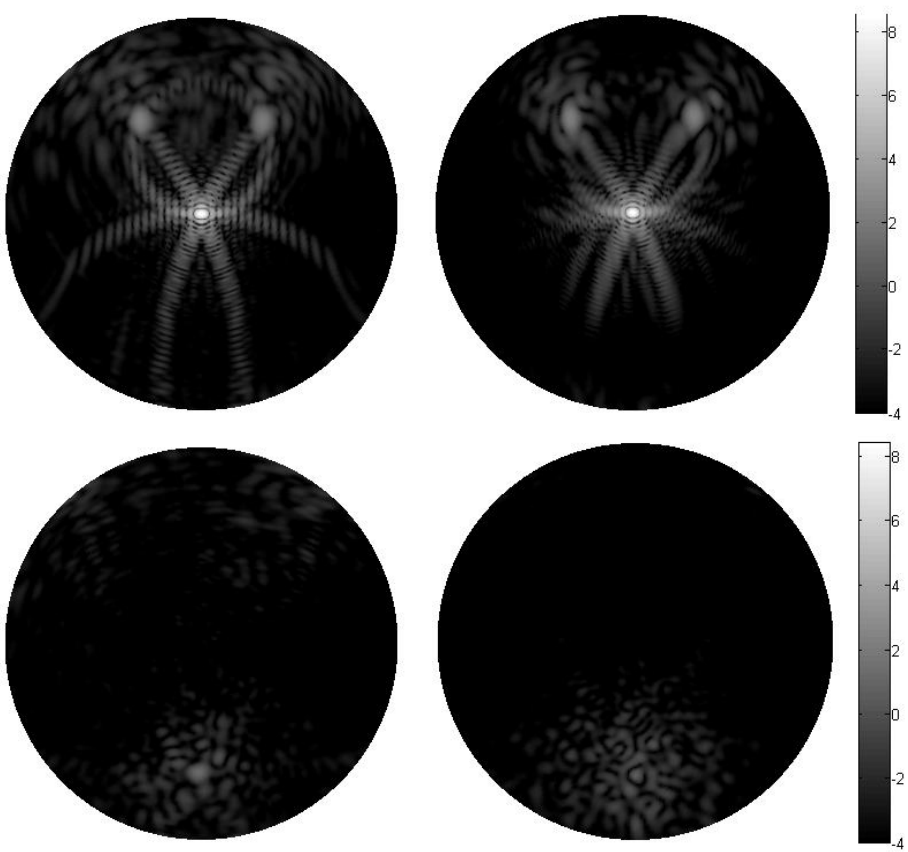

Fig. 11. 2D scattering patterns computed by DDA (right column) and beam tracing (left column) into forward and backscattering hemisphere (top and bottom row, respectively) for a hexagonal prism with Gaussian random surface (correlation length $0.5 \mu \mathrm{m}$, standard deviation $0.1 \mu \mathrm{m}$, average basal subfacet dimension is $0.412 \lambda$ ). 


\section{Summary}

The method of modelling diffraction by absorbing facetted particles [14] has been improved by implementing the vectorial representation of diffraction fields derived by Karczewski and Wolf using Kirchhoff type boundary conditions [17]. The method has been tested against DDA for a smooth hexagonal prism. For phase functions and azimuthally resolved scattering patterns good agreement with DDA results has been found, apart from slightly increased side scattering. The latter is most likely due to the modelling of diffraction as occurring at facets (i.e. the corresponding apertures) within infinite target planes and therefore not taking full account of the three-dimensionality of the scattering object. Analysis of the spatial distribution of the degree of linear polarisation illustrates the importance of adding external diffraction and reflection amplitudes, taking care of phase relations, rather than just adding intensities as is usually the case in Geometric Optics methods. Initial investigations into extending the method to facetted particles with slightly rough surface by introducing a surface scaling factor have been carried out. For a hexagonal prism with a Gaussian random surface, azimuthally resolved phase functions obtained by beam tracing and DDA show qualitatively similar speckle patterns.

\section{Acknowledgement}

E. Hesse, C.T Collier and Z. Ulanowski acknowledge support by the Natural Environment Research Council, United Kingdom (Grants no. NE/1020067/1 and C001350 (C.T.C.)). T. Nousiainen acknowledges funding by the Academy of Finland Grant no. 255718 and the Finnish Funding Agency for Technology and Innovation (Tekes) Grant no. 3155/31/2009. A. Penttilä acknowledges funding by the Academy of Finland Grant no. 1257966. The DDA results have been computed using CSC's resources. CSC is the Finnish IT Centre for Science and is owned by the Ministry of Education, Finland.

\section{References:}

[1] Baran A. A review of the light scattering properties of cirrus. Journal of Quantitative Spectroscopy and Radiative Transfer 2009;110:1239-60.

[2] Yu H, Kaufman YJ, Chin M, Feingold G, Remer LA, Anderson TL, et al. A review of measurement-based assessments of the aerosol direct radiative effect and forcing. Atmospheric Chemistry and Physics 2006;6:613-66.

[3] Lawson RP, Korolev AV, Cober SG, Huang T, Strapp JW, Isaac GA. Improved measurements of the drop size distribution of a freezing drizzle event. Atmospheric Research 1998;48:181-91.

[4] Kaye PH, Hirst E, Greenaway RS, Ulanowski Z, HesseE, DeMott PJ, Saunders C, Conolly P. Classifying atmospheric ice crystals by spatial light scattering. Optics Letters 2008;33:1545-7.

[5] Ulanowski Z, Kaye PK, Hirst E, Greenaway RS, Cotton RJ, Hesse E, and Collier C. Incidence of rough and irregular atmospheric ice particles from Small Ice Detector 3 measurements. Atmos. Chem. Phys. 2014;14:1649-1662.

[6] Mishchenko MI, Zakharova NT, Khlebtsov NG, Wriedt T, Videen G. Comprehensive thematic T-matrix reference database: A 2013-2014 update. J. Quant. Spectrosc. Radiat. Transfer 2014;146, 349-354.

[7] Yurkin MA, Maltsev VP, Hoekstra AG. The discrete dipole approximation: An overview and recent developments. Journal of Quantitative Spectroscopy and Radiative Transfer 2007;106:558-89.

[8] Yang P, Liou KN. In: Mishchenko MI, Hovenier JW, Travis LD, editors. Light scattering by nonspherical particles. New York: Academic Press; 1999. p. 173-221.

[9] Muinonen K. Scattering of light by crystals: a modified Kirchhoff approximation. Applied Optics 1989;28:3044-50. 
[10] Yang P, Liou KN. Geometric-optics-integral equation method for light scattering by nonspherical ice crystals. Applied Optics1996;35:6568-84.

[11] Bi L, Yang P, Kattawar GW, Hu Y, Baum BA. Scattering and absorption of light by ice particles: solution by a new physical-geometric optics hybrid method. Journal of Quantitative Spectroscopy and Radiative Transfer 2011:1492-508.

[12] Macke A, Mueller J, Raschke E. Single scattering properties of atmospheric ice crystals. Journal of the Atmospheric Sciences 1996;53:2813-25.

[13] Macke A, Mishchenko MI. Applicability of regular particle shapes in light scattering calculations for atmospheric ice particles. Applied optics 1996; 35(21): 4291-4296.

[14] Hesse E, Mc Call DS, Ulanowski Z, Stopford C, Kaye PH. Application of RTDF to particles with curved surfaces. Journal of Quantitative Spectroscopy and Radiative Transfer 2009:1599-603.

[15] Jackson JD. Classical electrodynamics. 3rd ed. New York: John Wiley \& Sons; 1999 [chapter 10.9].

[16] Hesse E, Macke A, Havemann S, Baran AJ, Ulanowski Z, Kaye PH. Modelling diffraction by facetted particles. J. Quantit. Spectrosc. Radiat. Transf. 2012:113, 342-347.

[17] Karczewski B, Wolf E. Comparison of three theories of electromagnetic diffraction at an aperture. Part I: Coherence Matrices. Journal of the Optical Society of America 1966;56:1207-1214.

[18] Shcherbakov V, Gayet J-F, Baker BA, Lawson RP. Light scattering by single natural ice crystals. J. Atmos. Sci. 2006, 63, 1513-1525.

[19] Baum BA, Yang P, Heymsfield AJ, Schmitt CG, Xie Y, Bansemer A, Hu YX, and Zhang Z. Improvements in shortwave bulk scattering and absorption models for the remote sensing of ice clouds, J. Appl. Meterol. Clim. 2011;50:1037-1056.

[20] Liu C, Panetta RL, Yang P. The effects of surface roughness on the scattering properties of hexagonal columns with sizes from the Rayleigh to the geometric optics regimes. J. Quantit. Spectrosc. Radiat. Transf. 2013; 129: 169-185.

[21] Baum, BA, Yang, P, Hu, YX, Feng, Q. The impact of ice particle roughness on the scattering phase matrix. Journal of Quantitative Spectroscopy and Radiative Transfer 2010;111:2534-49.

[22] Hovenier, JW, Mee, CVM. Fundamental relationships relevant to the transfer of polarized light in a scattering atmosphere. Astron. Astrophys. 1983;128:1-16.

[23] Born M, Wolf E. Principles of optics, 7th ed. Cambridge: CUP, 1999.

[24] Bohren H, Huffman DR. Absorption and scattering of light by small particles. New York: John Wiley \& Sons; 1998 [chapter 3.3].

[25] Kim SY, Vedam K. Analytic solution of the pseudo-Brewster angle. Journal of the Optical Society of America A 1986;3:1772-1773

[26] Muinonen K, Saarinen K. Ray Optics Approximation for Gaussian random cylinders. Journal of Quantitative Spectroscopy and Radiative Transfer 2000;64:201-18.

[27] Collier CT, Hesse E, Ulanowski Z, Penttilä A, Nousiainen T, Brousseau E, Hirshy H. Light scattering by Gaussian rough ice crystal analogues. P. 185 in Abstracts of conference 'Electromagnetic \& Light Scattering XIV' 17-21 June 2013 - Lille, France.

[28] Kahnert M, Nousiainen T, Mauno P. On the impact of non-sphericity and small-scale surface roughness on the optical properties of hematite aerosols. Journal of Quantitative Spectroscopy and Radiative Transfer 2011;112:1815-1824.

[29] Ulanowski Z, Hirst E, Kaye PH, Greenaway R. Retrieving the size of particles with rough and complex surfaces from two-dimensional scattering patterns. Journal of Quantitative Spectroscopy and Radiative Transfer 2012;113:2457-64.

[30] Yang P and Liou KN. Single-scattering properties of complex ice crystals in terrestrial atmosphere. Contr. Atmos. Phys. 1998;71:223-248.

[31] Clarke AJM, Hesse E, Ulanowski Z, and Kaye PH. A 3D implementation of ray tracing combined with diffraction on facets: Verification and a potential application. Journal of Quantitative Spectroscopy and Radiative Transfer 2006;100:103-114.

[32] Ogilvy JA. Accuracy of Kirchhoff theory. Chapter 4.2 in: Theory of wave scattering from random rough surfaces. Bristol: Adam Hilger, 1991. 